\title{
Total Synthesis of the Marine Natural Product (-)-Clavosolide A
}

\author{
Conor S. Barry, ${ }^{\dagger}$ Jon D. Elsworth, ${ }^{\dagger}$ Peter T. Seden, ${ }^{\dagger}$ Nick Bushby, ${ }^{\ddagger}$ \\ John R. Harding, ${ }^{\ddagger}$ Roger W. Alder, ${ }^{\dagger}$ and Christine L. Willis ${ }^{\star, \dagger}$ \\ ${ }^{\dagger}$ School of Chemistry, University of Bristol, Cantock's Close, Bristol, BS8 ITS, UK \\ ${ }^{*}$ AstraZeneca UK Ltd., Mereside, Alderley Park, Macclesfield, SK10 4TG, UK \\ Chris.Willis@bristol.ac.uk
}

\section{SUPPORTING INFORMATION}

\section{General Experimental Details}

All commercially available compounds were used without further purification except where stated. All moisture or air sensitive reactions were carried out in oven-dried glassware under a positive pressure of nitrogen using standard syringe/septa techniques. Anhydrous solvents were obtained by passing through a modified Grubbs system of alumina columns, manufactured by Anhydrous Engineering. When stated, DMF was dried sequentially over three portions of $10 \% \mathrm{w} / \mathrm{v} 3 \AA \mathrm{MS}$ beads for $24 \mathrm{~h}$ each, and then stored under nitrogen. Petroleum ether is of the $40-60{ }^{\circ} \mathrm{C}$ boiling point range. Routine monitoring of reactions was performed using precoated Merck-Keiselgel $60 \mathrm{~F}_{254}$ aluminium backed TLC plates. The spots were visualised by $\mathrm{UV}_{254}$ light, or potassium permanganate. Flash column chromatography ${ }^{1}$ was performed using silica gel (obtained from Fluorochem Ltd.) as the adsorbent.

Melting points were determined on an electrothermal apparatus and are uncorrected. Optical rotations were recorded using with the sodium D line $(\lambda=589 \mathrm{~nm})$ on a Bellingham and Stanley ADP220 polarimeter and the $[\alpha]_{\mathrm{D}}$ values are quoted in units $10^{-1} \mathrm{deg} \mathrm{cm}^{2} \mathrm{~g}^{-1}$. Infrared spectra were recorded on a Perkin Elmer Spectrum One FT-IR spectrometer in the solid or liquid state. ${ }^{1} \mathrm{H}$ and ${ }^{13} \mathrm{C}$ NMR spectra were recorded at using either a Jeol Delta/GX $400 \mathrm{MHz}$ or a Jeol Eclipse $400 \mathrm{MHz}$ spectrometer. The chemical shifts $(\delta)$ are reported in parts per million $(\mathrm{ppm})$ and the coupling constants $(J)$ are in Hertz $(\mathrm{Hz})$. Tetramethylsilane was used as the internal reference for proton and carbon chemical shifts. DEPT 135, COSY, HBQC and HMBC NMR spectra were routinely used to definitively assign the signals of ${ }^{1} \mathrm{H}$ and ${ }^{13} \mathrm{C}$ NMR spectra. Electron impact $(\mathrm{EI})$ and chemical ionisation $(\mathrm{CI})$ mass spectra were recorded on a VG Analytical Autospec mass spectrometer. Electrospray (ESI) mass spectra were recorded on a Micromass LCT mass spectrometer or a VG Quattro mass spectrometer. Methane was the ionisation gas used for chemical ionisation. Conformational searches were carried out in MMFF94 force field ${ }^{2}$ in Spartan. $^{3}$ 


\footnotetext{
${ }^{1}$ Still, W. C.; Kahn, M.; Mitra, A. J. Org. Chem. 1978, 43, 2923.

${ }^{2}$ Halgren, T. A. J. Computational Chem., 1996, 17, 490.

${ }^{3}$ Spartan '04, Wavefunction Inc., Irvine, CA, USA.
}

\section{Experimental Procedures}

\section{(+)-Methyl (2E, 5S, 7E)-5-(2-benzyloxyethyl)-4-oxa-non-2,7-dienoate 5}

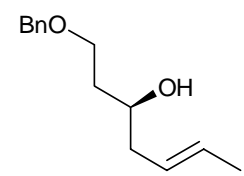

4

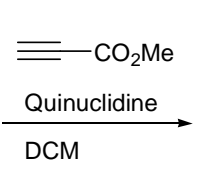

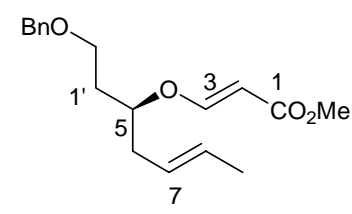

5

A solution of methyl propiolate $(6.10 \mathrm{ml}, 68.4 \mathrm{mmol})$ in dry DCM $(55 \mathrm{ml})$ was added dropwise over $7 \mathrm{~h}$ to a solution of alcohol 4 (13.71 g, $62.2 \mathrm{mmol})$ and quinuclidine $(211 \mathrm{mg}, 1.9 \mathrm{mmol})$ in DCM (70 ml) at room temperature under a nitrogen atmosphere. Upon complete addition, the reaction mixture was added to $5 \% \mathrm{v} / \mathrm{v} \mathrm{AcOH}_{\mathrm{aq}}(125 \mathrm{ml}, 0.35 \mathrm{M})$. The layers were separated and the aqueous phase was extracted with $\mathrm{Et}_{2} \mathrm{O}(4 \mathrm{x} 100 \mathrm{ml})$. The combined organic extracts were washed with saturated aqueous sodium hydrogen carbonate solution $(100 \mathrm{ml})$, dried $\left(\mathrm{MgSO}_{4}\right)$, filtered and concentrated in vacuo to give a brown oil (19.87 g) which was purified by column chromatography on silica gel, eluting with $12 \%$ EtOAc/petrol to give enol ether 5 (16.64 g, 88\%) as a colorless oil: silica gel TLC $R f 0.30$ (15\%

EtOAc/petrol); $[\alpha]_{\mathrm{D}}^{23}+56.6\left(c 4.4, \mathrm{CHCl}_{3}\right) ; v_{\max }($ neat $) / \mathrm{cm}^{-1} 3028(\mathrm{CH}), 2919(\mathrm{CH}), 2858(\mathrm{CH}), 1713$ $(\mathrm{C}=\mathrm{O}), 1640$ and $1622(\mathrm{C}=\mathrm{C}), 1454,1437,1202,1139 ; \delta_{\mathrm{H}}\left(400 \mathrm{MHz}, \mathrm{CDCl}_{3}\right) 1.65(3 \mathrm{H}, \mathrm{dd}, J 6.3,1.2$, 9- $\left.\mathrm{H}_{3}\right), 1.78-1.95\left(2 \mathrm{H}, \mathrm{m}, 1^{\prime}-\mathrm{H}_{2}\right), 2.25-2.31\left(2 \mathrm{H}, \mathrm{m}, 6-\mathrm{H}_{2}\right), 3.46-3.55\left(2 \mathrm{H}, \mathrm{m}, 2^{\prime}-\mathrm{H}_{2}\right), 3.69(3 \mathrm{H}, \mathrm{s},-\mathrm{OMe})$, $4.14(1 \mathrm{H}, \mathrm{m}, 5-\mathrm{H}), 4.47\left(2 \mathrm{H}, \mathrm{s},-\mathrm{CH}_{2} \mathrm{Ph}\right), 5.27(1 \mathrm{H}, \mathrm{d}, J 12.2,2-\mathrm{H}), 5.35(1 \mathrm{H}, \mathrm{dtq}, J 15.2,6.9,1.2,7-$ H), $5.50\left(1 \mathrm{H}, \mathrm{dq}, J\right.$ 15.2, 6.3, 8-H), 7.25-7.37 (5H, m, 5 x Ar-H), $7.53(1 \mathrm{H}, \mathrm{d}, J 12.2,3-\mathrm{H}) ; \delta_{\mathrm{C}}(100$ $\left.\mathrm{MHz}, \mathrm{CDCl}_{3}\right) 18.3$ (C-9), 36.5 (C-1'), 38.1 (C-6), 51.3 (-OMe), 66.2 (C-2'), $73.5\left(\mathrm{CH}_{2} \mathrm{Ph}\right), 81.1(\mathrm{C}-5)$,

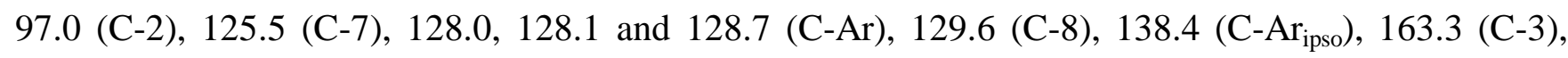
168.9 (C-1); Found (CI): $305.1753\left[\mathrm{MH}^{+},\left(\mathrm{C}_{18} \mathrm{H}_{25} \mathrm{O}_{4}\right.\right.$ requires 305.1751); m/z (CI) $305\left([\mathrm{MH}]^{+}, 20\right)$, $273\left([-\mathrm{Me}]^{+}, 23\right), 203\left(\left[-\mathrm{C}_{4} \mathrm{H}_{5} \mathrm{O}_{3}\right]^{+}, 57\right)$. Note: In some reactions the by-product, dimethyl $E$-hex-2-en-4yne-1,6-dioate 8, was also recovered: $\delta_{\mathrm{H}}\left(400 \mathrm{MHz}, \mathrm{CDCl}_{3}\right) 3.79(3 \mathrm{H}, \mathrm{s}, 6-\mathrm{OMe}), 3.83(3 \mathrm{H}, \mathrm{s}, 1-\mathrm{OMe})$, 
$6.48(1 \mathrm{H}, \mathrm{d}, J 16.1,4-\mathrm{H}), 6.79(1 \mathrm{H}, \mathrm{d}, J 16.1,5-\mathrm{H}) ; \delta_{\mathrm{C}}\left(100 \mathrm{MHz}, \mathrm{CDCl}_{3}\right) 52.3$ (6-OMe), $53.0(1-$ OMe), 81.7 (C-3), 86.7 (C-2), 121.7 (C-4), 135.0 (C-5), 153.5 (C-1), 165.1 (C-6).

(+)-Methyl $\quad(3 S, \quad 4 S, \quad 5 S, \quad 7 R, \quad 9 S, \quad 10 E)$-[7-(2-hydroxypent-3-enyl)-4-methyl-5(triisopropylsilanyloxy)-tetrahydropyran-2-yl]-acetate 10 and (+)-Methyl $\quad(3 S, \quad 4 S, \quad 5 S, \quad 7 R, \quad 9 R, \quad 10 E)$-[7-(2-hydroxypent-3-enyl)-4-methyl-5(triisopropylsilanyloxy)-tetrahydropyran-2-yl]-acetate 11

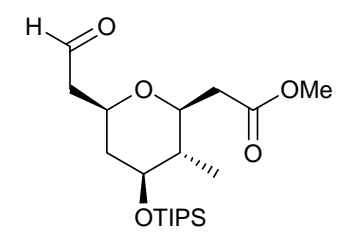

9

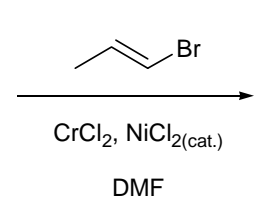

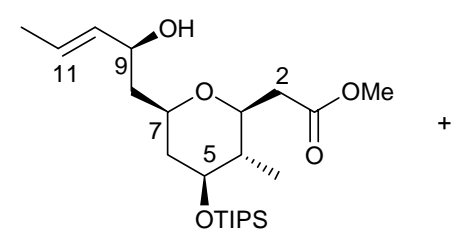

10

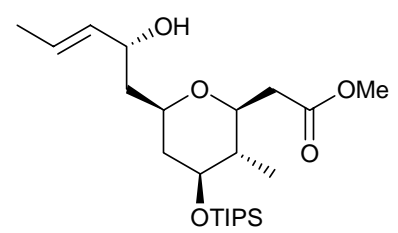

$\backslash 11$

Chromium (II) chloride (9.10 g, $74.04 \mathrm{mmol})$ and nickel (II) chloride (110 mg, $0.85 \mathrm{mmol})$ were added to a solution of aldehyde $9(5.70 \mathrm{~g}, 14.65 \mathrm{mmol})$ and $E$-1-bromo-1-propene $(2.60 \mathrm{ml}, 30.26 \mathrm{mmol})$ in dry DMF $(150 \mathrm{ml})$ at $0{ }^{\circ} \mathrm{C}$ under an argon atmosphere. The reaction mixture was stirred at $0{ }^{\circ} \mathrm{C}$ for $3 \mathrm{~h}$ and then at room temperature for $20 \mathrm{~h}$. The reaction was quenched by addition of saturated aqueous ammonium chloride solution $(250 \mathrm{ml})$. The mixture was stirred vigorously for $30 \mathrm{~min}$. and then added to saturated aqueous potassium sodium tartarate solution $(250 \mathrm{ml})$ and $\mathrm{Et}_{2} \mathrm{O}(300 \mathrm{ml})$. The layers were separated and the aqueous phase was extracted with $\mathrm{Et}_{2} \mathrm{O}(4 \times 300 \mathrm{ml})$. The combined organic extracts were washed with water $(2 \times 300 \mathrm{ml})$ and brine $(300 \mathrm{ml})$, dried $\left(\mathrm{MgSO}_{4}\right)$, filtered and concentrated in vacuo to give a pale green oil which was purified by column chromatography on silica gel, eluting with $30 \% \mathrm{Et}_{2} \mathrm{O} /$ hexane to give alcohol 10 (lower-running diastereomer by TLC) as a colorless oil (2.41 g, $39 \%)$ and alcohol 11 (higher-running diastereomer by TLC) as a colorless oil (2.29 g, 36\%): Data for 10; silica gel TLC Rf 0.24 (20\% EtOAc/petrol); $[\alpha]^{23}{ }_{\mathrm{D}}+13.3\left(c\right.$ 1.2, $\left.\mathrm{CHCl}_{3}\right) ; v_{\max }$ (neat) $/ \mathrm{cm}^{-1} 3526$, 2945, 2892 and $2867(\mathrm{CH}), 1744(\mathrm{C}=\mathrm{O}), 1464,1438,1092 ; \delta_{\mathrm{H}}\left(400 \mathrm{MHz}, \mathrm{CDCl}_{3}\right) 0.98(3 \mathrm{H}, \mathrm{d}, J$ 6.6, 4- $\left.\mathrm{CH}_{3}\right), 1.02-1.08(3 \mathrm{H}, \mathrm{m}, 3 \times \mathrm{Si}-\mathrm{CH}), 1.06\left(18 \mathrm{H}, \mathrm{s}, 3 \times \mathrm{SiCH}\left(\mathrm{CH}_{3}\right)_{2}\right), 1.34(1 \mathrm{H}, \mathrm{tq}, J$ 9.8, 6.5, 4-H), $1.43\left(1 \mathrm{H}, \mathrm{dt}, J 12.6,11.2,6-\mathrm{H}_{\mathrm{ax}}\right), 1.56(1 \mathrm{H}, \mathrm{dt}, J 14.5,2.6,8-H \mathrm{H}), 1.67\left(3 \mathrm{H}, \mathrm{dd}, J 6.5,1.4,12-\mathrm{H}_{3}\right)$, $1.74(1 \mathrm{H}, \mathrm{dt}, J 14.5,10.0,8-\mathrm{H} H), 1.87$ (1H, ddd, $\left.J 12.6,4.6,1.7,6-\mathrm{H}_{\mathrm{eq}}\right), 2.41(1 \mathrm{H}, \mathrm{dd}, J 14.9,10.0,2-$ $H \mathrm{H}), 2.67(1 \mathrm{H}, \mathrm{dd}, J 14.9,2.7,2-\mathrm{HH}), 3.39(1 \mathrm{H}, \mathrm{br} \mathrm{s},-\mathrm{OH}), 3.47-3.65(3 \mathrm{H}, \mathrm{m}, 3-\mathrm{H}, 5-\mathrm{H}$ and $7-\mathrm{H})$, 
$3.70(3 \mathrm{H}, \mathrm{s},-\mathrm{OMe}), 4.26(1 \mathrm{H}, \mathrm{m}, 9-\mathrm{H}), 5.45(1 \mathrm{H}, \mathrm{ddq}, J 15.2,6.8,1.4,10-\mathrm{H}), 5.67$ (1H, dqd, $J 15.2$, 6.5, 0.7, 11-H); $\delta_{\mathrm{C}}\left(100 \mathrm{MHz}, \mathrm{CDCl}_{3}\right) 12.8(3 \mathrm{x} \mathrm{SiCH}), 13.2\left(4-\mathrm{CH}_{3}\right), 17.6(\mathrm{C}-12), 18.1$ and $18.3(3 \mathrm{x}$ $\left.\mathrm{SiCH}\left(\mathrm{CH}_{3}\right)_{2}\right), 38.7$ (C-2), 42.1 (C-6), 43.0 (C-8), 44.1 (C-4), 51.9 (-OMe), 72.7 (C-9), 73.7 (C-5), 76.1 (C-7), 78.1 (C-3), 126.1 (C-11), 133.4 (C-10), 172.2 (C-1); Found (CI): $429.3024[\mathrm{MH}]^{+},\left(\mathrm{C}_{23} \mathrm{H}_{45} \mathrm{O}_{5} \mathrm{Si}\right.$ requires, 429.3036); $m / z(\mathrm{CI}) 429\left([\mathrm{MH}]^{+}, 5\right), 411\left(\left[\mathrm{M}^{+}-\mathrm{OH}\right], 62\right), 385\left(\left[\mathrm{M}^{+}{ }_{-}^{\mathrm{i}} \mathrm{Pr}\right], 96\right), 299(100), 237$ (49), 219 (90). Data for 11: silica gel TLC $R f 0.28$ (20\% EtOAc/petrol); $[\alpha]_{\mathrm{D}}^{23}+16.7\left(c 1.2, \mathrm{CHCl}_{3}\right)$; $v_{\max }($ neat $) / \mathrm{cm}^{-1} 3483,2945,2892$ and $2867(\mathrm{CH}), 1744(\mathrm{C}=\mathrm{O}), 1686,1464,1438 ; \delta_{\mathrm{H}}(400 \mathrm{MHz}$, $\left.\mathrm{CDCl}_{3}\right) 0.98\left(3 \mathrm{H}, \mathrm{d}, J\right.$ 6.6, 4- $\left.\mathrm{CH}_{3}\right), 1.03-1.09$ (3H, m, 3 x Si-CH), $1.06\left(18 \mathrm{H}, \mathrm{s}, 3\right.$ x SiCH$\left.\left(\mathrm{CH}_{3}\right)_{2}\right), 1.34$ $(1 \mathrm{H}, \mathrm{tq}, J 9.9,6.6,4-\mathrm{H}), 1.45\left(1 \mathrm{H}, \mathrm{dt}, J 12.3,10.5,6-\mathrm{H}_{\mathrm{ax}}\right), 1.60(1 \mathrm{H}, \mathrm{ddd}, J 14.4,7.6,3.2,8-H \mathrm{H}), 1.70$ $\left(3 \mathrm{H}, \mathrm{dd}, J 6.5,1.5,12-\mathrm{H}_{3}\right), 1.73-1.80(1 \mathrm{H}, \mathrm{m}, 8-\mathrm{H} H), 1.84\left(1 \mathrm{H}, \mathrm{ddd}, J 12.3,4.6,1.5,6-\mathrm{H}_{\mathrm{eq}}\right), 2.41(1 \mathrm{H}$, dd, $J 14.9,9.9,2-H \mathrm{H}), 2.66(1 \mathrm{H}, \mathrm{dd}, J 14.9,3.1,2-\mathrm{H} H), 2.94(1 \mathrm{H}, \mathrm{d}, J 6.1,-\mathrm{OH}), 3.48(1 \mathrm{H}, \mathrm{td}, J 9.9$, 3.1, 3-H), 3.55 (1H, ddd, J 10.5, 9.9, 4.6, 5-H), 3.63-3.70 (1H, m, 7-H), $3.70(3 \mathrm{H}, \mathrm{s},-\mathrm{OMe}), 4.26(1 \mathrm{H}$, m, 9-H), $5.50(1 \mathrm{H}, \mathrm{ddq}, J 15.3,6.2,1.5,10-\mathrm{H}), 5.67(1 \mathrm{H}, \mathrm{dqd}, J 15.3,6.5,1.1,11-\mathrm{H}) ; \delta_{\mathrm{C}}(100 \mathrm{MHz}$ $\left.\mathrm{CDCl}_{3}\right) 12.8(3 \times \mathrm{SiCH}), 13.3\left(4-\mathrm{CH}_{3}\right), 17.7(\mathrm{C}-12), 18.1$ and $18.3\left(3 \times \mathrm{SiCH}\left(\mathrm{CH}_{3}\right)_{2}\right), 38.9(\mathrm{C}-2), 41.7$ (C-6), 42.0 (C-8), 44.2 (C-4), 51.8 (-OMe), 69.6 (C-9), 72.8 (C-7), 74.1 (C-5), 78.1 (C-3), 125.7 (C11), 133.5 (C-10), 172.3 (C-1); Found (CI): $429.3021[\mathrm{MH}]^{+},\left(\mathrm{C}_{23} \mathrm{H}_{45} \mathrm{O}_{5} \mathrm{Si}\right.$ requires, 429.3036); m/z

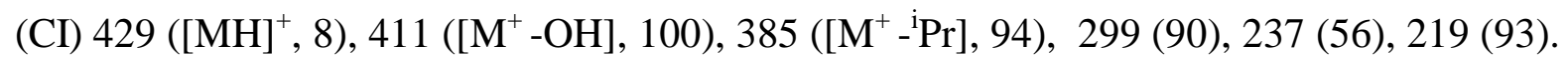

\section{(+)-Methyl (3S, 4S, 5S, 7R, 10E)-[7-(2-oxopent-3-enyl)-4-methyl-5-(triisopropylsilanyloxy)-} tetrahydropyran-2-yl]-acetate 11a

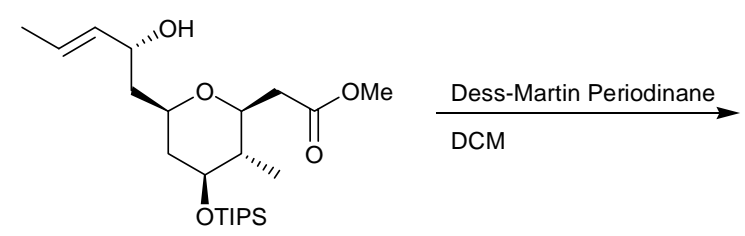

11

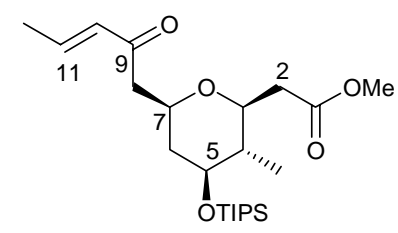

11a

A solution of Dess-Martin periodinone (15\% v/v in DCM, $1.5 \mathrm{eq}, 0.79 \mathrm{mmol}, 1.65 \mathrm{ml})$ was added to a stirred solution of alcohol $11(0.53 \mathrm{mmol}, 227 \mathrm{mg})$ in dry DCM $(10 \mathrm{ml})$ at RT under a nitrogen atmosphere. The mixture was stirred for $2 \mathrm{~h}$ and quenched by the addition of saturated aqueous sodium hydrogen carbonate $(25 \mathrm{ml})$ and DCM $(25 \mathrm{ml})$. The layers were separated and the aqueous fraction was extracted with DCM $(40 \mathrm{ml})$. The combined organic fractions were washed with water $(25 \mathrm{ml})$ and brine $(25 \mathrm{ml})$, dried $\left(\mathrm{MgSO}_{4}\right)$, filtered and concentrated in vacuo to give a yellow oil which was purified by column chromatography on silica gel, eluting with $10 \%$ EtOAc in petrol to give enone 11a $(223 \mathrm{mg}, 99 \%)$ as a pale yellow oil: silica gel TLC $R f 0.35\left(20 \%\right.$ EtOAc/petrol); $[\alpha]^{23}{ }_{\mathrm{D}}+16.7$ (c 1.2 , $\left.\mathrm{CH}_{2} \mathrm{Cl}_{2}\right) ; v_{\max }$ (neat)/cm ${ }^{-1}$ 2944, 2892 and $2866(\mathrm{CH}), 1742$ and $1672(\mathrm{C}=\mathrm{O}), 1631(\mathrm{C}=\mathrm{C}), 1087,1065$; 


\section{Supporting Information}

$\delta_{\mathrm{H}}\left(400 \mathrm{MHz}, \mathrm{CDCl}_{3}\right) 0.97\left(3 \mathrm{H}, \mathrm{d}, J\right.$ 6.6, 4- $\left.\mathrm{CH}_{3}\right), 1.01-1.08(3 \mathrm{H}, \mathrm{m}, 3$ x SiCH), $1.06(18 \mathrm{H}, \mathrm{s}, 3$ x $\left.\mathrm{SiCH}\left(\mathrm{CH}_{3}\right)_{2}\right), 1.32\left(1 \mathrm{H}, \mathrm{tq}, J\right.$ 9.8, 6.6, 4-H), $1.34\left(1 \mathrm{H}, \mathrm{dt}, J 12.5,11.5,6-\mathrm{H}_{\mathrm{ax}}\right), 1.90(3 \mathrm{H}, \mathrm{dd}, J 6.8,1.2$, 12- $\left.\mathrm{H}_{3}\right), 1.99\left(1 \mathrm{H}, \mathrm{ddd}, J 12.5,4.6,1.7,6-\mathrm{H}_{\mathrm{eq}}\right), 2.36(1 \mathrm{H}, \mathrm{dd}, J 14.7,9.5,2-H \mathrm{H}), 2.48(1 \mathrm{H}, \mathrm{dd}, J 15.3$, 5.4, 8-HH), $2.62(1 \mathrm{H}, \mathrm{dd}, J 14.7,3.4,2-\mathrm{H} H), 2.86(1 \mathrm{H}, \mathrm{dd}, J 15.3,7.2,8-\mathrm{H} H), 3.48(1 \mathrm{H}, \mathrm{td}, J$ 9.8, 3.4, 3-H), 3.56 (1H, ddd, J 11.5, 9.8, 4.6, 5-H), 3.63 (3H, s, OMe), 3.82 (1H, m 7-H), 6.11 (1H, dq, J 15.6, 1.2, 10-H), $6.83\left(1 \mathrm{H}, \mathrm{dq}, J\right.$ 15.6, 6.8, 11-H); $\delta_{\mathrm{C}}\left(100 \mathrm{MHz}, \mathrm{CDCl}_{3}\right) 12.9\left(3\right.$ x SiCH), $13.3\left(4-\mathrm{CH}_{3}\right), 18.2$ and 18.3 (3 x $\left.\operatorname{SiCH}\left(\mathrm{CH}_{3}\right)_{2}\right), 39.4$ (C-2), 41.8 (C-6), 44.3 (C-4), 46.0 (C-8), $51.6(\mathrm{OMe}), 72.2$ (C-7), 74.0 (C-5), 78.1 (C-3), 132.5 (C-10), 143.5 (C-11), 172.0 (C-1), 198.2 (C-9); Found (ESI): 449.2686 $[\mathrm{MNa}]^{+},\left(\mathrm{C}_{23} \mathrm{H}_{42} \mathrm{O}_{5} \mathrm{Si}\right.$ requires 449.2694$) ; \mathrm{m} / z(\mathrm{ESI}) 449\left([\mathrm{MNa}]^{+}, 100 \%\right)$.

\section{Luche Reduction}

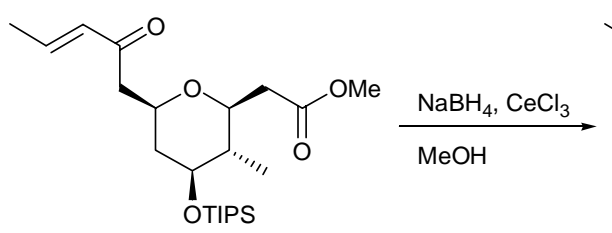

$11 a$

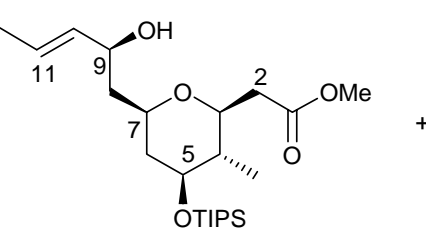

10

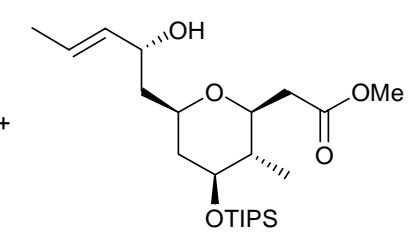

11

Sodium borohydride ( $1 \mathrm{eq}, 0.26 \mathrm{mmol}, 9.9 \mathrm{mg}$ ) was added in small portions to a stirred solution of enone $11 \mathrm{a}(0.26 \mathrm{mmol}, 112 \mathrm{mg})$ and cerium trichloride heptahydrate $(0.26 \mathrm{mmol}, 97.8 \mathrm{mg})$ at $0{ }^{\circ} \mathrm{C}$ in $\mathrm{MeOH}(8 \mathrm{ml})$. The mixture was stirred for $3 \mathrm{~h}$ and the volatiles removed in vacuo to give a yellow precipitate which was purified by column chromatography on silica gel, eluting with $20 \%$ EtOAc in petrol to give a 2:1 (11:10) mixture of diastereomers $(101 \mathrm{mg}, 90 \%)$ as a colorless oil: Spectroscopic data as above.

(+)-Methyl (3S, 4S, 5S, 7R, 9S, 10E)-[7-(2-(tert-butyldimethylsilanyloxy)-pent-3-enyl)-4-methyl-5(triisopropylsilanyloxy)-tetrahydropyran-2-yl]-acetate 10a

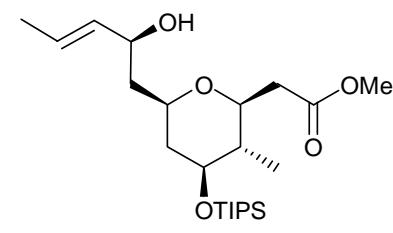

10

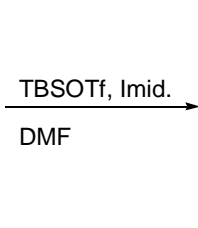

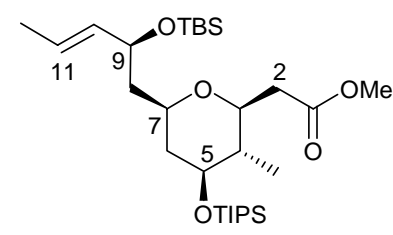

$10 \mathbf{a}$ 


\section{Supporting Information}

tert-Butyldimethylsilyltriflate $(0.177 \mathrm{ml}, 0.77 \mathrm{mmol})$ was added to a solution of imidazole $(72 \mathrm{mg}$, $1.05 \mathrm{mmol})$ and alcohol $10(300 \mathrm{mg}, 0.70 \mathrm{mmol})$ in dry DMF (5 ml) at room temperature under a nitrogen atmosphere. The mixture was stirred for $19 \mathrm{~h}$ and then water $(25 \mathrm{ml})$ and $\mathrm{Et}_{2} \mathrm{O}(25 \mathrm{ml})$ were added. The layers were separated and the aqueous phase was extracted with $\mathrm{Et}_{2} \mathrm{O}(3 \mathrm{x} 25 \mathrm{ml})$. The combined organic extracts were washed with water $(2 \times 25 \mathrm{ml})$ and brine $(25 \mathrm{ml})$, dried $\left(\mathrm{MgSO}_{4}\right)$, filtered and concentrated in vacuo to give a colorless oil which was purified by flash column chromatography on silica gel, eluting with $5 \% \mathrm{Et}_{2} \mathrm{O} /$ hexanes to give alkene 10a (376 $\left.\mathrm{mg}, 92 \%\right)$ as a colorless oil: silica gel TLC $R f 0.63$ (20\% EtOAc/petrol); $[\alpha]_{\mathrm{D}}^{25}+21.0\left(c 2.0, \mathrm{CHCl}_{3}\right) ; v_{\max }(\mathrm{neat}) / \mathrm{cm}^{-1}$ 2948, 2892 and $2867(\mathrm{CH}), 1747(\mathrm{C}=\mathrm{O}), 1464,1437 ; \delta_{\mathrm{H}}\left(400 \mathrm{MHz}, \mathrm{CDCl}_{3}\right) 0.01$ (3H, s, SiMe), 0.02 (3H, s, SiMe), $0.86\left(9 \mathrm{H}, \mathrm{s}, 3\right.$ x Si $\left.\left(\mathrm{CH}_{3}\right)_{3}\right), 0.95$ (3H, d, J 6.6, 4- $\left.\mathrm{CH}_{3}\right), 1.01-1.07$ (3H, m, 3 x $\left.\mathrm{SiCH}\right), 1.05$ $\left(18 \mathrm{H}, \mathrm{s}, 3 \times \mathrm{SiCH}\left(\mathrm{CH}_{3}\right)_{2}\right), 1.30\left(1 \mathrm{H}, \mathrm{tq}, J\right.$ 9.8, 6.6, 4-H), $1.34\left(1 \mathrm{H}, \mathrm{dt}, J 12.5,11.5,6-\mathrm{H}_{\mathrm{ax}}\right), 1.42(1 \mathrm{H}$, ddd, $J$ 13.3, 8.0, 4.0, 8- HH), $1.68\left(3 \mathrm{H}, \mathrm{dd}, J 6.4,1.5,12-\mathrm{H}_{3}\right), 1.78(1 \mathrm{H}, \mathrm{ddd}, J 13.3,9.1,4.9,8-\mathrm{H} H)$, $1.85\left(1 \mathrm{H}, \mathrm{ddd}, J 12.5,4.6,1.7,6-\mathrm{H}_{\mathrm{eq}}\right), 2.38(1 \mathrm{H}, \mathrm{dd}, J 14.7,10.0,2-H \mathrm{H}), 2.61(1 \mathrm{H}, \mathrm{dd}, J 14.7,3.2,2-$ HH), 3.27 (1H, dddd, $J$ 11.0, 9.1, 4.0, 1.7, 7-H), 3.39 (1H, td, $J$ 9.8, 3.2, 3-H), 3.49 (1H, ddd, $J 11.5$, 9.8, 4.6, 5-H), 3.68 (3H, s, OMe), 4.17 (1H, td, J 8.0, 4.9, 9-H), 5.34 (1H, ddq, J 15.4, 8.0, 1.5, 10-H), $5.52(1 \mathrm{H}, \mathrm{dqd}, J 15.4,6.4,0.7,11-\mathrm{H}) ; \delta_{\mathrm{C}}\left(100 \mathrm{MHz}, \mathrm{CDCl}_{3}\right)-4.9(\mathrm{SiMe}),-4.3(\mathrm{SiMe}), 12.8(3 \times \mathrm{SiCH})$, $13.3\left(4-\mathrm{CH}_{3}\right), 17.7(\mathrm{C}-12), 18.2$ and $\left.18.3\left(3 \mathrm{x} \mathrm{SiCH}\left(\mathrm{CH}_{3}\right)_{2}\right)\right), 25.9\left(\mathrm{SiC}(\mathrm{CH})_{3}\right), 39.2(\mathrm{C}-2), 41.9(\mathrm{C}-6)$, 44.4 (C-4), 44.5 (C-8), 51.5 (OMe), 70.8 (C-9), 72.1 (C-7), 74.3 (C-5), 77.8 (C-3), 125.9 (C-11), 134.0 (C-10), 172.3 (C-1); Found (ESI): 565.3696 [MNa] ${ }^{+},\left(\mathrm{C}_{29} \mathrm{H}_{58} \mathrm{O}_{5} \mathrm{Si}_{2} \mathrm{Na}\right.$ requires, 565.3715); $m / z$ (ESI) $565\left([\mathrm{MNa}]^{+}, 100\right)$.

\section{(+)-Methyl (3S, 4S, 5S, 7R, 9S, 10R, 11R)-9-(tert-butyldimethylsilanyloxy)-4-methyl-5-}

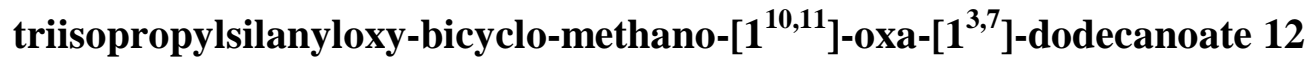

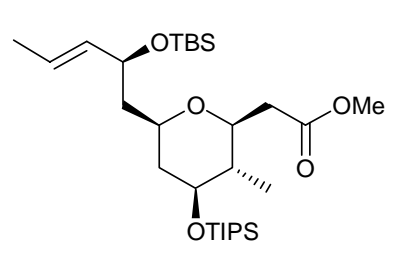

$10 a$

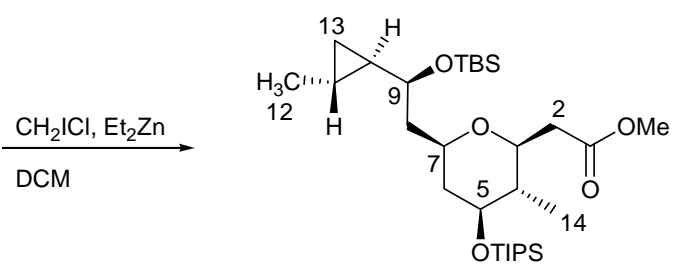

12

Chloroiodomethane (10 eq, $9.10 \mathrm{mmol}, 663 \mu \mathrm{L})$ was added to a solution of diethyl zinc (5 eq, $1.0 \mathrm{M}$ in hexanes, $4.55 \mathrm{mmol}, 4.55 \mathrm{ml})$ in dry DCM $(12 \mathrm{ml})$ at $0{ }^{\circ} \mathrm{C}$ under a nitrogen atmosphere. The mixture was stirred for $0.5 \mathrm{~h}$ (white ppt forms) and then a solution of alkene 10a $(0.91 \mathrm{mmol}, 495 \mathrm{mg})$ in dry DCM (6 ml) was added via syringe followed by dry DCM washings $(5 \mathrm{ml})$. The mixture was stirred for 


\section{Supporting Information}

$5 \mathrm{~h}$ slowly warming to $15{ }^{\circ} \mathrm{C}$ and was then quenched by addition to saturated aqueous ammonium chloride solution $(120 \mathrm{ml})$. $\mathrm{Et}_{2} \mathrm{O}(70 \mathrm{ml})$ was added, the layers were separated and the aqueous phase was extracted with $\mathrm{Et}_{2} \mathrm{O}(2 \times 70 \mathrm{ml})$. The combined organic extracts were washed with brine $(70 \mathrm{ml})$, dried $\left(\mathrm{MgSO}_{4}\right)$, filtered and concentrated in vacuo to give a slightly opaque oil which was purified by flash column chromatography on silica gel, eluting with $5 \% \mathrm{Et}_{2} \mathrm{O} /$ hexanes to give cyclopropane 12 $(366 \mathrm{mg}, 72 \%)$ as a colorless oil: silica gel TLC $R f 0.48\left(10 \%\right.$ EtOAc/petrol); $[\alpha]_{\mathrm{D}}^{25}+22.0$ (c 2.0, $\left.\mathrm{CHCl}_{3}\right) ; v_{\max }\left(\right.$ neat)$/ \mathrm{cm}^{-1} 2948,2893$ and $2866(\mathrm{C}-\mathrm{H}), 1747(\mathrm{C}=\mathrm{O}) ; \delta_{\mathrm{H}}\left(400 \mathrm{MHz}, \mathrm{CDCl}_{3}\right) 0.01(3 \mathrm{H}, \mathrm{s}$, SiMe), 0.03 (3H, s, SiMe), 0.17 (1H, dt, J 8.1, 4.6, 13-HH), 0.37 (1H, dt, J 8.1, 4.6, 13-HH), 0.50-0.60 $\left(2 \mathrm{H}, \mathrm{m}, 10-\mathrm{H}\right.$ and 11-H), $0.87\left(9 \mathrm{H}, \mathrm{s}, 3\right.$ x Si $\left.\left(\mathrm{CH}_{3}\right)_{3}\right), 0.97\left(3 \mathrm{H}, \mathrm{d}, J\right.$ 6.6, 14- $\left.\mathrm{H}_{3}\right), 1.02(3 \mathrm{H}, \mathrm{d}, J 5.6,12-$ $\left.\mathrm{H}_{3}\right), 1.06\left(21 \mathrm{H}, \mathrm{s}, 3 \mathrm{x} \mathrm{Si}-\mathrm{CH}\right.$ and $\left.3 \mathrm{x} \mathrm{SiCH}\left(\mathrm{CH}_{3}\right)_{2}\right), 1.25-1.37\left(2 \mathrm{H}, \mathrm{m}, 4-\mathrm{H}\right.$ and 6- $\left.\mathrm{H}_{\mathrm{ax}}\right), 1.55(1 \mathrm{H}, \mathrm{ddd}, J$ 13.4, 8.1, 4.0, 8-HH), $1.78(1 \mathrm{H}, \mathrm{ddd}, J 13.4,9.1,4.7,8-\mathrm{H} H), 1.87\left(1 \mathrm{H}, \mathrm{ddd}, J 12.2,4.4,1.7,6-\mathrm{H}_{\mathrm{eq}}\right.$ ), $2.37(1 \mathrm{H}, \mathrm{dd}, J 14.6,9.9,2-H \mathrm{H}), 2.61$ (1H, dd, $J$ 14.6, 3.0, 2-HH), 3.14 (1H, td, $J$ 8.1, 4.7, 9-H), 3.41 $(1 \mathrm{H}, \mathrm{td}, J 9.9,3.0,3-\mathrm{H}), 3.47-3.57\left(2 \mathrm{H}, \mathrm{m}, 5-\mathrm{H}\right.$ and 7-H), $3.65(3 \mathrm{H}, \mathrm{s}, \mathrm{OMe}) ; \delta_{\mathrm{C}}\left(100 \mathrm{MHz}, \mathrm{CDCl}_{3}\right)-$ 4.6 (SiMe), -4.3 (SiMe), 10.7 (C-13), 10.9 (C-11), 12.8 (3 x SiCH), 13.4 (C-14), $18.1\left(\mathrm{SiC}_{-}\left(\mathrm{CH}_{3}\right)_{3}\right)$, 18.2 and $18.3\left(3 \times \operatorname{SiCH}\left(\mathrm{CH}_{3}\right)_{2}\right), 18.7(\mathrm{C}-12), 25.7(\mathrm{C}-10), 25.9\left(\mathrm{SiC}-(\mathrm{CH})_{3}\right), 39.3(\mathrm{C}-2), 42.3(\mathrm{C}-6)$, 44.4 (C-4), 45.1 (C-8), 51.5 (OMe), 72.2 (C-5 or C-7), 73.2 (C-9), 74.3 (C-5 or C-7), 77.8 (C-3), 172.3 (C-1); Found (ESI): $579.3878[\mathrm{MNa}]^{+},\left(\mathrm{C}_{30} \mathrm{H}_{60} \mathrm{O}_{5} \mathrm{Si}_{2} \mathrm{Na}\right.$ requires, 579.3871); m/z (ESI) $579\left([\mathrm{MNa}]^{+}\right.$, 100).

(+)-Methyl $(3 S, 4 S, 5 S, 7 R, 9 S, 10 R, 11 R)-9$-hydroxy-4-methyl-5-triisopropylsilanyloxy-bicyclomethano-[1 $\left.1^{10,11}\right]$-oxa-[1 $\left.1^{3,7}\right]$-dodecanoate 13

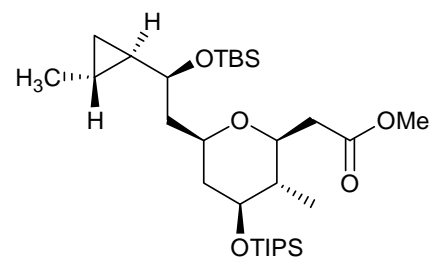

12

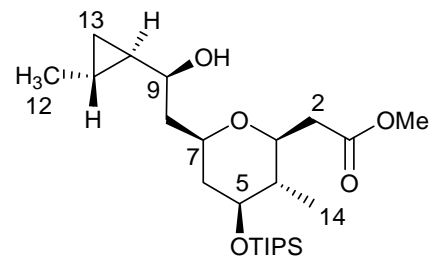

13

$12 \mathrm{M} \mathrm{HCl}_{\mathrm{aq}}(5 \mathrm{eq}, 3.14 \mathrm{mmol}, 260 \mu \mathrm{L})$ was mixed with $95 \% \mathrm{EtOH}(14 \mathrm{ml})$ and added to a vigorously stirring solution of cyclopropane $12(0.63 \mathrm{mmol}, 350 \mathrm{mg})$ in $95 \% \mathrm{EtOH}(12 \mathrm{ml})$. The mixture was stirred at room temperature for $50 \mathrm{~min}$ and then added to saturated aqueous sodium hydrogen carbonate solution $(160 \mathrm{ml})$ and DCM $(160 \mathrm{ml})$. The layers were separated and the aqueous phase was extracted with DCM (2 x $120 \mathrm{ml})$. The combined organic extracts were washed with water $(140 \mathrm{ml})$ and brine $(140 \mathrm{ml})$, dried $\left(\mathrm{MgSO}_{4}\right)$, filtered and concentrated in vacuo to give a pale yellow oil which was 


\section{Supporting Information}

purified by flash column chromatography on silica gel, eluting with $40 \% \mathrm{Et}_{2} \mathrm{O}$ /petrol to give alcohol 13 $(262 \mathrm{mg}, 94 \%)$ as a colorless oil: silica gel TLC $\operatorname{Rf} 0.28\left(40 \%\right.$ EtOAc/petrol); $[\alpha]_{\mathrm{D}}^{25}+4.5$ (c 2.7 , $\left.\mathrm{CHCl}_{3}\right) ; v_{\max }($ neat $) / \mathrm{cm}^{-1} 3530(\mathrm{OH}), 2946,2893$ and $2867(\mathrm{C}-\mathrm{H}), 1743(\mathrm{C}=\mathrm{O}) ; \delta_{\mathrm{H}}\left(400 \mathrm{MHz}, \mathrm{CDCl}_{3}\right)$ $0.15(1 \mathrm{H}, \mathrm{dt}, J 8.4,4.6,13-H \mathrm{H}), 0.30(1 \mathrm{H}, \mathrm{dt}, J 8.4,4.6,13-\mathrm{H} H), 0.58(1 \mathrm{H}, \mathrm{tt}, J$ 8.4, 4.6, 10-HH), 0.77 $(1 \mathrm{H}, \mathrm{m}, 11-\mathrm{H}), 0.97\left(3 \mathrm{H}, \mathrm{d}, J\right.$ 6.6, 14- $\left.\mathrm{H}_{3}\right), 1.05\left(3 \mathrm{H}, \mathrm{d}, J\right.$ 6.1, 12- $\left.\mathrm{H}_{3}\right), 1.06(21 \mathrm{H}, \mathrm{s}, 3 \mathrm{x}$ Si-CH and 3 x $\left.\operatorname{SiCH}\left(\mathrm{CH}_{3}\right)_{2}\right), 1.34\left(1 \mathrm{H}, \mathrm{tq}, J\right.$ 9.8, 6.6, 4-H), $1.42\left(1 \mathrm{H}, \mathrm{dt}, J 12.3,11.2,6-\mathrm{H}_{\mathrm{ax}}\right), 1.65(1 \mathrm{H}, \mathrm{dt}, J 14.5,2.7$, 8-HH), $1.75(1 \mathrm{H}, \mathrm{dt}, J 14.5,9.6,8-\mathrm{H} H), 1.87\left(1 \mathrm{H}, \mathrm{ddd}, J 12.3,4.6,1.8,6-\mathrm{H}_{\mathrm{eq}}\right), 2.40(1 \mathrm{H}, \mathrm{dd}, J 15.2$, 9.8, 2-HH), $2.65(1 \mathrm{H}, \mathrm{dd}, J$ 15.2, 2.8, 2-HH), $3.11(1 \mathrm{H}, \mathrm{td}, J$ 8.4, 2.7, 9-H), 3.49 (1H, td, $J$ 9.8, 2.8, 3$\mathrm{H}), 3.48-3.60\left(2 \mathrm{H}, \mathrm{m}, 5-\mathrm{H}\right.$ and 7-H), $3.68(3 \mathrm{H}, \mathrm{s}, \mathrm{OMe}) ; \delta_{\mathrm{C}}\left(100 \mathrm{MHz}, \mathrm{CDCl}_{3}\right) 10.0(\mathrm{C}-13), 11.2(\mathrm{C}-$ 11), $12.8(\mathrm{SiCH}), 13.3(\mathrm{C}-14), 18.2$ and $18.3\left(\mathrm{SiCH}\left(\mathrm{CH}_{3}\right)_{2}\right), 18.7(\mathrm{C}-12), 26.1(\mathrm{C}-10), 38.6(\mathrm{C}-2), 42.2$ (C-6), 42.6 (C-8), 44.0 (C-4), 51.9 (OMe), 73.8 and 76.1 (C-5 and C-7), 76.3 (C-9), 77.9 (C-3), 172.1 (C-1); Found (ESI): $465.3006[\mathrm{MNa}]^{+},\left(\mathrm{C}_{30} \mathrm{H}_{60} \mathrm{O}_{5} \mathrm{Si}_{2} \mathrm{Na}\right.$ requires, 465.3007$) ; \mathrm{m} / z$ (ESI) $465\left([\mathrm{MNa}]^{+}\right.$, 100).

\section{(-)-(3S,4S,5S,7R,9S,10R,11R)-9-Hydroxy-4-methyl-5-triisopropylsilanyloxy-bicyclo-methano-} $\left[1^{10,11}\right]$-oxa-[1 $\left.1^{3,7}\right]$-dodecanoic acid 14

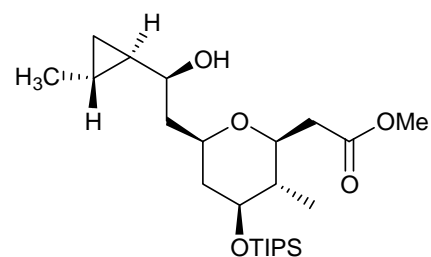

13

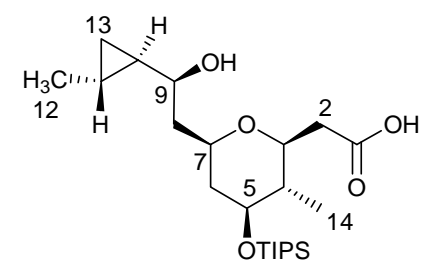

14

Sodium trimethylsilanolate (1M in DCM, $2 \mathrm{eq}, 1.45 \mathrm{mmol}, 1.45 \mathrm{ml}$ ) was added to a stirred solution of ester $13(0.72 \mathrm{mmol}, 320 \mathrm{mg})$ in dry DCM $(18 \mathrm{ml})$ at RT under a nitrogen atmosphere. The mixture was stirred for $18 \mathrm{~h}$ and then the volatiles were removed in vacuo to give the sodium salt as a pale yellow solid which was purified by column chromatography on silica gel, eluting with 67\% EtOAc in petrol followed by $67 \%$ EtOAc in petrol $+4 \% \mathrm{AcOH}$ to give acid 14 (307 $\mathrm{mg}, 99 \%)$ as a pale yellow oil: silica gel TLC $R f 0.61\left(67 \%\right.$ EtOAc/petrol + 4\% AcOH); $[\alpha]^{24}{ }_{\mathrm{D}}-5.6\left(c 5.0, \mathrm{CH}_{2} \mathrm{Cl}_{2}\right) ; v_{\max }(\mathrm{neat}) / \mathrm{cm}^{-}$ ${ }^{1} 3456(\mathrm{OH}), 2943,2893$ and $2866(\mathrm{CH}), 1715,1463 ; \delta_{\mathrm{H}}\left(400 \mathrm{MHz}, \mathrm{CDCl}_{3}\right) 0.17(1 \mathrm{H}, \mathrm{dt}, J 8.5$ and 5.0, 13-HH), $0.27(1 \mathrm{H}, \mathrm{dt}, J 8.5$ and 5.0, 13-HH), $0.59(1 \mathrm{H}, \mathrm{tt}, J 8.5$ and 5.0, 10-H), $0.77(1 \mathrm{H}, \mathrm{m}, 11-$ H), $0.98\left(3 \mathrm{H}, \mathrm{d}, J\right.$ 7.0, 14- $\left.\mathrm{H}_{3}\right), 1.02\left(3 \mathrm{H}, \mathrm{d}, J 5.0,12-\mathrm{H}_{3}\right), 1.03-1.08(3 \mathrm{H}, \mathrm{m}, 3$ x SiCH), $1.06(18 \mathrm{H}, \mathrm{s}, 3$ x $\left.\mathrm{SiCH}\left(\mathrm{CH}_{3}\right)_{2}\right), 1.34(1 \mathrm{H}, \mathrm{m}, 4-\mathrm{H}), 1.44\left(1 \mathrm{H}, \mathrm{dt}, J 12.0\right.$ and 11.5, 6- $\left.\mathrm{H}_{\mathrm{ax}}\right), 1.67(1 \mathrm{H}, \mathrm{br} \mathrm{d}, J 14.0,8-H \mathrm{H})$, $1.79(1 \mathrm{H}, \mathrm{m}, 8-\mathrm{H} H), 1.88\left(1 \mathrm{H}, \mathrm{ddd}, J 12.0,5.0\right.$ and 1.5, 6- $\left.\mathrm{H}_{\mathrm{eq}}\right), 2.40(1 \mathrm{H}, \mathrm{dd}, J 15.0$ and 11.0, 2-HH), $2.66(1 \mathrm{H}, \mathrm{dd}, J 15.0$ and 2.0, 2-HH), $3.16(1 \mathrm{H}, \mathrm{dt}, J 8.5$ and 2.0, 9-H), 3.46-3.62 (3H, m, 3-H, 5-H and 
7-H); $\delta_{\mathrm{C}}\left(100 \mathrm{MHz}, \mathrm{CDCl}_{3}\right) 10.2(\mathrm{C}-13), 11.7(\mathrm{C}-11), 12.8$ (3 x SiCH), $13.3(\mathrm{C}-14), 18.2$ and 18.3 (3 x $\left.\mathrm{SiCH}\left(\mathrm{CH}_{3}\right)_{2}\right), 18.6$ (C-12), 25.8 (C-10), 38.6 (C-2), 41.8 (C-6), 42.1 (C-8), 44.0 (C-4), 73.7 and 76.5 (C-5 and C-7), 77.2 (C-9), 78.2 (C-3), 173.4 (C-1); Found (ESI): 451.2856 [MNa] $^{+},\left(\mathrm{C}_{23} \mathrm{H}_{44} \mathrm{O}_{5} \mathrm{Si}\right.$ requires 451.2850$) ; m / z(\mathrm{ESI}) 451\left([\mathrm{MNa}]^{+}, 100 \%\right)$.

\section{(-)-(3S,4S,5S,7R,9S,10R,11R,3'S,4'S,5' $S, 7$ ' $R, 9$ ' $S, 10 ' R, 11 ' R)-4,4$ '-Dimethyl-9,9'-bis-(2-} methylcyclopropyl)-5,5'-triisopropylsilanyloxy-15,16,15',16'-tetraoxytricyclo[15.3.1.1 $\left.{ }^{3,7}\right]$ docosane-1,1'-dione $14 a$

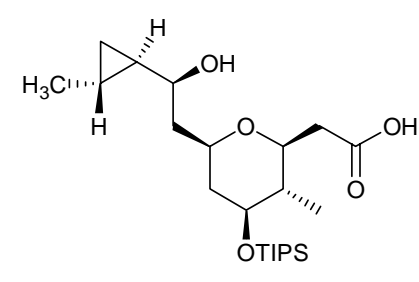

14
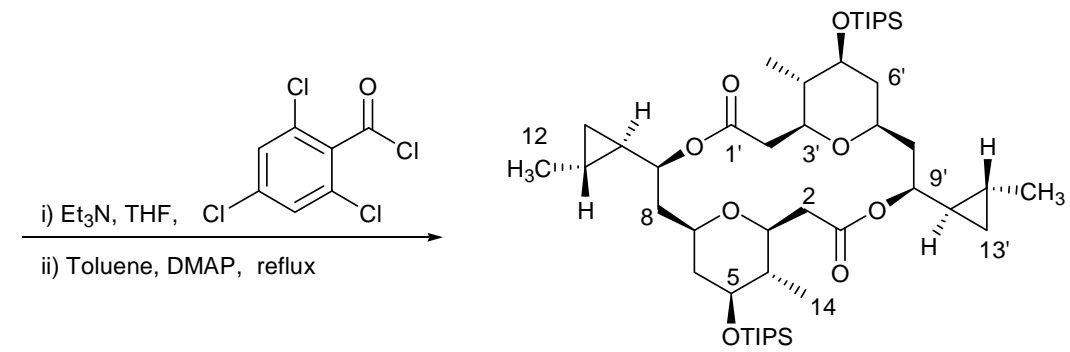

$14 a$

2,4,6-Trichlorobenzoyl chloride (1.1 eq, $0.44 \mathrm{mmol}, 68.2 \mu \mathrm{L})$ was added to a stirred solution of secoacid $14(0.40 \mathrm{mmol}, 170 \mathrm{mg})$ and triethylamine $(1.3 \mathrm{eq}, 0.52 \mathrm{mmol}, 71.9 \mu \mathrm{L})$ in dry THF $(1.4 \mathrm{ml})$ at RT under a nitrogen atmosphere. The mixture was stirred for $2.5 \mathrm{~h}$. The TEA.HCl salt was filtered off and the filtrate diluted with dry toluene $(15 \mathrm{ml})$. This solution was then added slowly to a solution of dimethylaminopyridine $(5.1 \mathrm{eq}, 2.02 \mathrm{mmol}, 247 \mathrm{mg})$ in dry toluene $(70 \mathrm{ml})$ under reflux. The mixture was stirred for a further $17 \mathrm{~h}$ and diluted with water (75) at RT. The layers were separated and the aqueous layer was extracted with $\mathrm{Et}_{2} \mathrm{O}(2 \times 35 \mathrm{ml})$. The combined organic fractions washed with brine $(50 \mathrm{ml})$, dried $\left(\mathrm{MgSO}_{4}\right)$, filtered and concentrated in vacuo to give a white solid which was purified by flash column chromatography on silica gel, eluting with $10 \% \mathrm{Et}_{2} \mathrm{O}$ in hexane to give dimer 14a (121 $\mathrm{mg}, 74 \%$ ) as a white solid (which could not be recrystallised): silica gel TLC Rf 0.75 (50\%

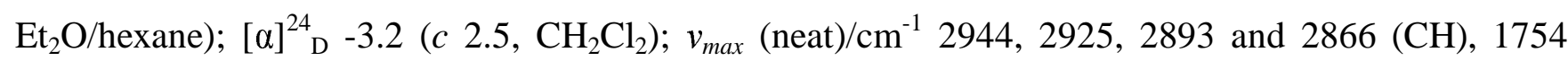
$(\mathrm{C}=\mathrm{O}), 1464,1054 ; \delta_{\mathrm{H}}\left(400 \mathrm{MHz}, \mathrm{CDCl}_{3}\right) 0.23(2 \mathrm{H}, \mathrm{dt}, J$ 8.6, 4.6, 13-HH and 13'-HH), $0.36(2 \mathrm{H}, \mathrm{dt}, J$ 


\section{Supporting Information}

8.6, 4.6, 13-H H and 13'-HH), 0.69 (2H, tt, $J$ 8.6, 4.6, 10-H and 10'-H), 0.79 (2H, m, 11-H and 11'-H), $0.92\left(6 \mathrm{H}, \mathrm{d}, J 6.6,14-\mathrm{H}_{3}\right.$ and $\left.14^{\prime}-\mathrm{H}_{3}\right), 0.97\left(6 \mathrm{H}, \mathrm{d}, J 5.9,12-\mathrm{H}_{3}\right.$ and 12'- $\left.\mathrm{H}_{3}\right), 1.02-1.09(6 \mathrm{H}, \mathrm{m}, 6 \mathrm{x}$ $\mathrm{SiCH}), 1.06\left(36 \mathrm{H}, \mathrm{s}, 6 \times \mathrm{SiCH}\left(\mathrm{CH}_{3}\right)_{2}\right), 1.27\left(2 \mathrm{H}, \mathrm{m}, 4-\mathrm{H}\right.$ and 4'-H), $1.32\left(2 \mathrm{H}, \mathrm{dt}, J, 12.0,11.5,6-\mathrm{H}_{\mathrm{ax}}\right.$ and 6'- $\left.\mathrm{H}_{\mathrm{ax}}\right), 1.65\left(2 \mathrm{H}\right.$, dt, $J 14.9,2.0,8-H \mathrm{H}$ and $\left.8^{\prime}-H \mathrm{H}\right), 1.75\left(2 \mathrm{H}\right.$, ddd, $J 12.0,4.9,1.7,6-\mathrm{H}_{\mathrm{eq}}$ and 6'$\left.\mathrm{H}_{\mathrm{eq}}\right), 1.88\left(2 \mathrm{H}, \mathrm{dt}, J 14.9,8.6,8-\mathrm{H} H\right.$ and $\left.8^{\prime}-\mathrm{H} H\right), 2.43(2 \mathrm{H}, \mathrm{dd}, J 17.8,5.6,2-H \mathrm{H}$ and 2-'HH), $2.54(2 \mathrm{H}$, dd, $J$ 17.8, 4.4, 2-H H and 2'-HH), 3.43-3.54 (6H, m, 3-H and 3'-H, 5-H and 5'-H, 7-H and 7'-H), 4.45 $\left(2 \mathrm{H}, \mathrm{td}, J 8.6,1.7,9-\mathrm{H}\right.$ and 9'-H); $\delta_{\mathrm{C}}\left(100 \mathrm{MHz}, \mathrm{CDCl}_{3}\right) 11.0\left(\mathrm{C}-11\right.$ and $\left.\mathrm{C}-11^{\prime}\right), 11.7$ (C-13 and C-13'), $12.8(\mathrm{SiCH}), 13.2\left(\mathrm{C}-14\right.$ and $\left.\mathrm{C}-144^{\prime}\right), 18.2$ and $18.3\left(\mathrm{SiCH}\left(\mathrm{CH}_{3}\right)_{2}\right), 18.5\left(\mathrm{C}-12\right.$ and $\left.\mathrm{C}-12^{\prime}\right), 24.7(\mathrm{C}-10$ and C-10'), 39.4 (C-2 and C-2'), 41.4 (C-8 and C-8'), 42.5 (C-6 and C-6'), 44.8 (C-4 and C-4'), 74.3 (C5 and C-5'), 74.9 (C-7 and C-7'), 77.0 (C-3 and C-3'), 77.2 (C-9 and C-9'), 171.2 (C-1 and C-1'); Found (ESI): $843.5614[\mathrm{MNa}]^{+},\left(\mathrm{C}_{46} \mathrm{H}_{84} \mathrm{O}_{8} \mathrm{Si}_{2} \mathrm{Na}\right.$ requires 843.5597); $\mathrm{m} / z$ (ESI) $843\left([\mathrm{MNa}]^{+}, 100\right)$.

\section{(-)-(3S,4S,5S,7R,9S,10R,11R,3' $\left.S, 4^{\prime} S, 5^{\prime} S, 7^{\prime} R, 9 ' S, 10 ' R, 11 ' R\right)-4,4^{\prime}$-Dimethyl-9,9'-bis-(2-methyl cyclopropyl)-5,5'-bis-hydroxy-15,16,15',16'-tetraoxy-tricyclo[15.3.1.1 $\left.{ }^{3,7}\right]$ docosane-1,1'-dione 15}

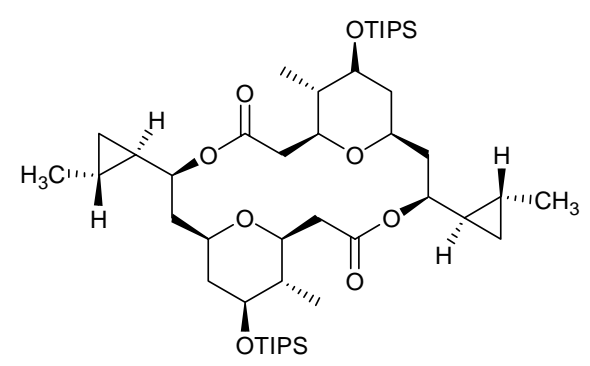

$14 a$

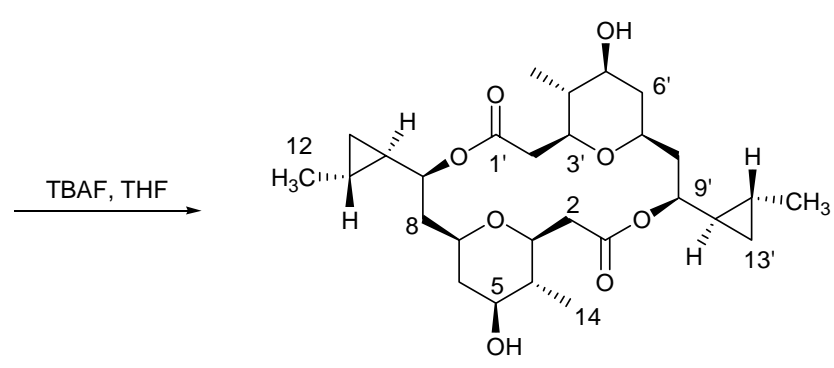

15

TBAF (8 eq, $1 \mathrm{M}$ in THF, $1.12 \mathrm{mmol}, 1.12 \mathrm{ml})$ was added to a solution of dimer $14 \mathbf{a}(0.115 \mathrm{mmol}, 121$ $\mathrm{mg}$ ) in dry THF (5 ml) at RT under a nitrogen atmosphere. The mixture was stirred for $3 \mathrm{~h}$ and then added to 1:1 water/EtOAc $(30 \mathrm{ml})$. The layers were separated and the aqueous phase was extracted with EtOAc $(3 \times 25 \mathrm{ml})$. The combined organic fractions were washed with water $(2 \times 25 \mathrm{ml})$ and brine $(25 \mathrm{ml})$, dried $\left(\mathrm{MgSO}_{4}\right)$, filtered and concentrated in vacuo to give a yellow oil which was purified by column chromatography on silica gel, eluting with 40\% EtOAc in petrol to give diolide 15 (54 mg, $72 \%$ ) as a white solid (which could not be recrystallised): silica gel TLC $R f 0.07$ (50\% $\mathrm{Et}_{2} \mathrm{O} / \mathrm{hexane}$ );

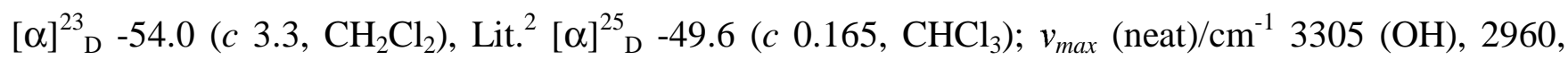
2920 and $2854(\mathrm{CH}), 1722(\mathrm{C}=\mathrm{O}), 1024 ; \delta_{\mathrm{H}}\left(400 \mathrm{MHz}, \mathrm{CDCl}_{3}\right) 0.24(2 \mathrm{H}, \mathrm{dt}, J$ 8.6, 4.6, 13-HH and $\left.13^{\prime}-H \mathrm{H}\right), 0.36(2 \mathrm{H}, \mathrm{dt}, J$ 8.6, 4.6, 13-H $H$ and 13'-HH), $0.73(2 \mathrm{H}, \mathrm{tt}, J 8.6,4.6,10-\mathrm{H}$ and 10'-H), 0.82 $\left(2 \mathrm{H}, \mathrm{m}, 11-\mathrm{H}\right.$ and $\left.11^{\prime}-\mathrm{H}\right), 0.95\left(6 \mathrm{H}, \mathrm{d}, J 6.6,14-\mathrm{H}_{3}\right.$ and $\left.14^{\prime}-\mathrm{H}_{3}\right), 0.98\left(6 \mathrm{H}, \mathrm{d}, J\right.$ 6.1, 12- $-\mathrm{H}_{3}$ and $\left.12^{\prime}-\mathrm{H}_{3}\right)$, $1.23(2 \mathrm{H}, \mathrm{m}, 4-\mathrm{H}$ and $4 '-\mathrm{H}), 1.26\left(2 \mathrm{H}, \mathrm{dt}, J, 12.0,11.5,6-\mathrm{H}_{\mathrm{ax}}\right.$ and $\left.6 '-\mathrm{H}_{\mathrm{ax}}\right), 1.70(2 \mathrm{H}, \mathrm{ddd}, J 15.1,3.4$, 


\section{Supporting Information}

2.0, 8- $-H \mathrm{H}$ and 8'-HH), 1.88-1.96 (4H, m, 6- $\mathrm{H}_{\mathrm{eq}}$ and 6'- $\mathrm{H}_{\mathrm{eq}}, 8-\mathrm{H} H$ and 8'-H $\left.H\right), 2.43(2 \mathrm{H}, \mathrm{dd}, J$ 17.1, 6.6, 2- $H \mathrm{H}$ and 2-' $H \mathrm{H}), 2.56\left(2 \mathrm{H}, \mathrm{dd}, J 17.1,3.7,2-\mathrm{H} H\right.$ and $\left.2^{\prime}-\mathrm{H} H\right), 3.36$ (2H, td, $J$ 11.5, 4.4, 5-H and 5'-H), 3.42 (2H, dt, $J$ 6.6, 3.7, 3-H and 3'-H), 3.49 (2H, m, 7-H and 7'-H), 4.42 (2H, td, J 8.6, 1.5, 9-H and 9'$\mathrm{H}) ; \delta_{\mathrm{C}}\left(100 \mathrm{MHz}, \mathrm{CDCl}_{3}\right) 10.9$ (C-13 and C-13'), 11.9 (C-11 and C-11'), 12.7 (C-14 and C-14'), 18.5 (C-12 and C-12'), 24.7 (C-10 and C-10'), 39.1 (C-2 and C-2'), 41.3 (C-8 and C-8'), 41.4 (C-6 and C-6'), 44.0 (C-4 and C-4'), 73.1 (C-5 and C-5'), 74.8 (C-7 and C-7'), 76.9 (C-9 and C-9'), 77.0 (C-3 and C3'), 171.1 (C-1 and C-1'); Found (ESI): $531.2934[\mathrm{MNa}]^{+},\left(\mathrm{C}_{28} \mathrm{H}_{44} \mathrm{O}_{8} \mathrm{Na}\right.$ requires 531.2928); $m / z$ (ESI) $531\left([\mathrm{MNa}]^{+}, 100\right)$.

${ }^{2}$ Son, J. B.; Kim, S. N.; Kim, N. Y.; Lee, D. H. Org. Lett., 2006, 8661.

(-)-(3S, 4S, 5S, 7R, 9S, 10R, 11R, 15S, 16R, 17S, 18R, 3'S, 4'S, 5'S, 7'R, 9'S, 10' $R, 1^{\prime} R, 1^{\prime} S, 16 ' R$, $17 ' S, 18 ' R)-4,4^{\prime}$-Dimethyl-9,9'-bis-(2-methylcyclopropyl)-5,5'-bis-(3,4,5-trimethoxy tetrahydropyran -2-yloxy)-15,16,15',16'-tetraoxa-tricyclo[15.3.1.1 $\left.{ }^{3,7}\right]$ docosane-1,1'-dione 2

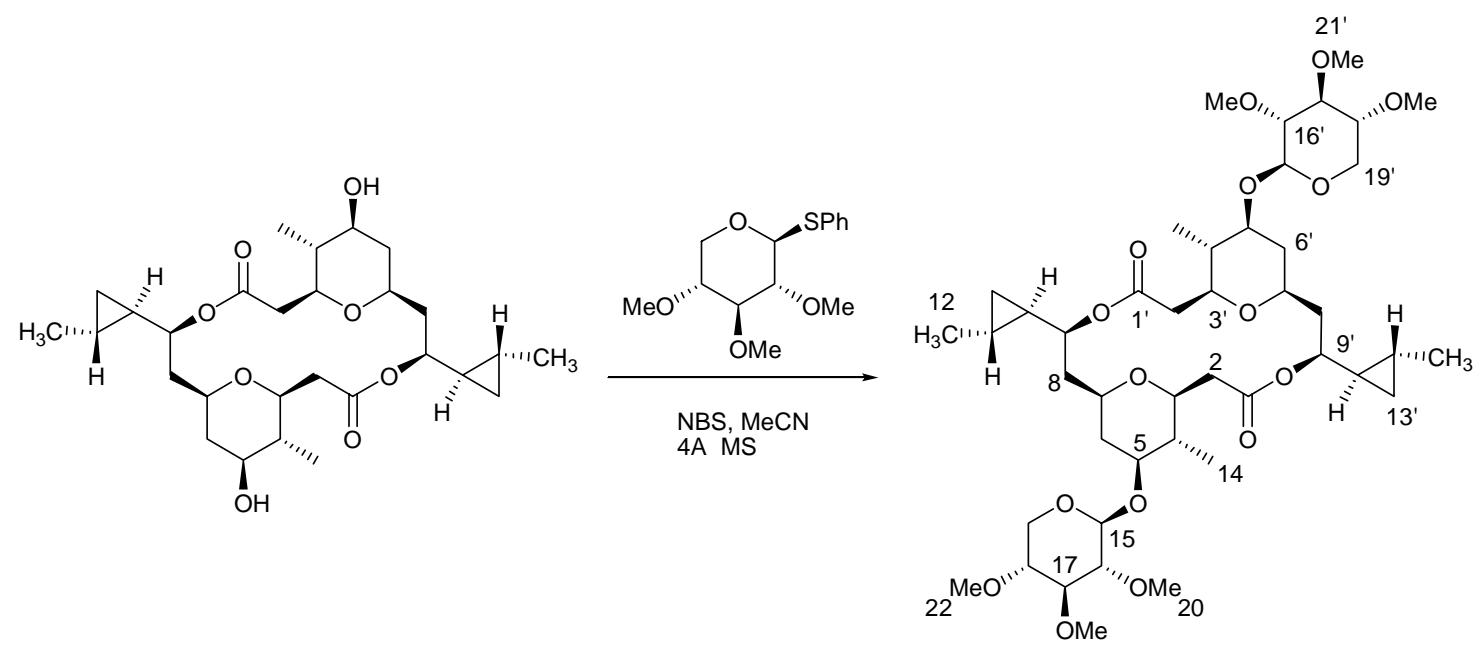

17

2

A solution of NBS (8 eq, $0.88 \mathrm{mmol}, 157 \mathrm{mg}$ ) in dry MeCN (3 ml) and washings (1 ml MeCN) was added slowly to a stirred solution of diolide $17(0.11 \mathrm{mmol}, 56 \mathrm{mg})$, thioglycoside (4 eq, $0.44 \mathrm{mmol}$, $126 \mathrm{mg})$ and activated powdered $4 \AA$ molecular sieves $(250 \mathrm{mg})$ in dry $\mathrm{MeCN}(3 \mathrm{ml})$ and washings $(2$ $\mathrm{ml}$ DCM) at -40 C under a nitrogen atmosphere. The mixture was stirred for $1 \mathrm{~h}$ slowly warming to RT (white $\rightarrow$ orange) and for a further $0.5 \mathrm{~h}$ at RT. The solution was then filtered into saturated aqueous sodium hydrogen carbonate solution $(25 \mathrm{ml})$ and diluted with EtOAc $(20 \mathrm{ml})$. The layers were 


\section{Supporting Information}

separated and the aqueous fraction extracted with EtOAc $(2 \times 20 \mathrm{ml})$. The combined organic fractions were washed with brine $(30 \mathrm{ml})$, dried $\left(\mathrm{MgSO}_{4}\right)$, filtered and concentrated in vacuo to give a pale yellow oil which was purified by column chromatography on silica gel, eluting with 60\% EtOAc in petrol to give diolide 2 (17 mg, 18\%) as a white solid: silica gel TLC $R f 0.43$ (60\% EtOAc/petrol); mp 252-254 ${ }^{\circ} \mathrm{C}$, Lit. $^{2} 256.8^{\circ} \mathrm{C} ;[\alpha]_{\mathrm{D}}^{23}-38.0\left(c\right.$ 1.0, $\left.\mathrm{CHCl}_{3}\right) ; v_{\max }(\mathrm{film}) / \mathrm{cm}^{-1} 2944(\mathrm{CH}), 1736(\mathrm{C}=\mathrm{O}), 1072$; $\delta_{\mathrm{H}}\left(400 \mathrm{MHz}, \mathrm{CDCl}_{3}\right) 0.22\left(2 \mathrm{H}, \mathrm{dt}, J 8.3,4.9,13-H \mathrm{H}\right.$ and $\left.13{ }^{\prime}-H \mathrm{H}\right), 0.34(2 \mathrm{H}, \mathrm{dt}, J$ 8.3, 4.9, 13-H $H$ and $13 '-\mathrm{H} H), 0.71(2 \mathrm{H}, \mathrm{tt}, J$ 8.3, 4.9, 10-H and 10'-H), $0.81(2 \mathrm{H}, \mathrm{m}, 11-\mathrm{H}$ and 11'-H), 0.97 (12H, d, J 6.5, $12-\mathrm{H}_{3}$ and $12^{\prime}-\mathrm{H}_{3}, 14-\mathrm{H}_{3}$ and $\left.14^{\prime}-\mathrm{H}_{3}\right), 1.37\left(2 \mathrm{H}, \mathrm{q}, J 11.5,6-\mathrm{H}_{\mathrm{ax}}\right.$ and 6'- $\left.\mathrm{H}_{\mathrm{ax}}\right), 1.38(2 \mathrm{H}, \mathrm{m}, 4-\mathrm{H}$ and 4'H), $1.68(2 \mathrm{H}$, br d, $J 15.1,8-H \mathrm{H}$ and 8'- $H \mathrm{H}), 1.89(2 \mathrm{H}, \mathrm{dt}, J 15.1,9.0,8-\mathrm{H} H$ and 8'-H $H), 2.05(2 \mathrm{H}$, ddd, $J 11.5,4.6,1.5,6-\mathrm{H}_{\mathrm{eq}}$ and $\left.6{ }^{\prime}-\mathrm{H}_{\mathrm{eq}}\right), 2.41\left(2 \mathrm{H}, \mathrm{dd}, J 17.3,6.6,2-H \mathrm{H}\right.$ and $\left.2^{\prime}-H \mathrm{H}\right), 2.55(2 \mathrm{H}, \mathrm{dd}, J$ 17.3, 3.7, 2-H $H$ and 2'-H $H$ ), 2.96 (2H, dd, $J$ 9.0, 7.8, 16-H and 16'-H), 3.09 (2H, dd, $J 11.2,10.0,19-$ $\mathrm{H}_{\mathrm{ax}}$ and $\left.19^{\prime}-\mathrm{H}_{\mathrm{ax}}\right), 3.10(2 \mathrm{H}, \mathrm{t}, J$ 9.0,17-H and 17'-H,) 3.21-3.28 (4H, m, 5-H and 5'-H, 18-H and 18'-H), 3.41-3.49 (4H, m, 3-H and 3'-H, 7-H and 7'-H), $3.47\left(6 \mathrm{H}, \mathrm{s}, 22-\mathrm{H}_{3}\right.$ and $\left.22^{\prime}-\mathrm{H}_{3}\right), 3.57\left(6 \mathrm{H}, \mathrm{s}, 20-\mathrm{H}_{3}\right.$ and $\left.20^{\prime}-\mathrm{H}_{3}\right), 3.61\left(6 \mathrm{H}, \mathrm{s}, 21-\mathrm{H}_{3}\right.$ and $\left.21^{\prime}-\mathrm{H}_{3}\right), 3.96\left(2 \mathrm{H}, \mathrm{dd}, J 11.2,5.1,19-\mathrm{H}_{\mathrm{eq}}\right.$ and $\left.19 '-\mathrm{H}_{\mathrm{eq}}\right), 4.27(2 \mathrm{H}, \mathrm{d}, J$ 7.8, 15-H and 15'-H), $4.41\left(2 \mathrm{H}, \mathrm{td}, J\right.$ 9.0, 2.0, 9-H and 9'-H); $\delta_{\mathrm{C}}\left(100 \mathrm{MHz}, \mathrm{CDCl}_{3}\right) 10.9(\mathrm{C}-13$ and C13'), 11.9 (C-11 and C-11'), 12.6 (C-14 and C-14'), 18.5 (C-12 and C-12'), 24.7 (C-10 and C-10'), 39.2 (C-2 and C-2'), 40.7 (C-6 and C-6'), 41.3 (C-8 and C-8'), 42.6 (C-4 and C-4'), 58.8 (C-22 and C-22'), 60.4 (C-20 and C-20'), 60.8 (C-21 and C-21'), 63.2 (C-19 and C-19'), 74.8 (C-7 and C-7'), 77.0 (C-3 and C-3'), 77.1 (C-9 and C-9'), 79.4 (C-18 and C-18'), 83.2 (C-5 and C-5'), 83.8 (C-16 and C-16'), 85.6 (C-17 and C-17'), 105.5 (C-15 and C-15'), 171.0 (C-1 and C-1'); Found (ESI): 879.4737 [MNa] $\left(\mathrm{C}_{44} \mathrm{H}_{72} \mathrm{O}_{16} \mathrm{Na}\right.$ requires 879.4713); $\mathrm{m} / z$ (ESI) $879\left([\mathrm{MNa}]^{+}, 100\right)$.

The ${ }^{1} \mathrm{H}$ - and ${ }^{13} \mathrm{C}$ NMR spectra of the aglycone $\mathbf{1 5}$ and synthetic clavosolide A $\mathbf{2}$ are given below. 
${ }^{1} \mathrm{H}$ Aglycone 15.
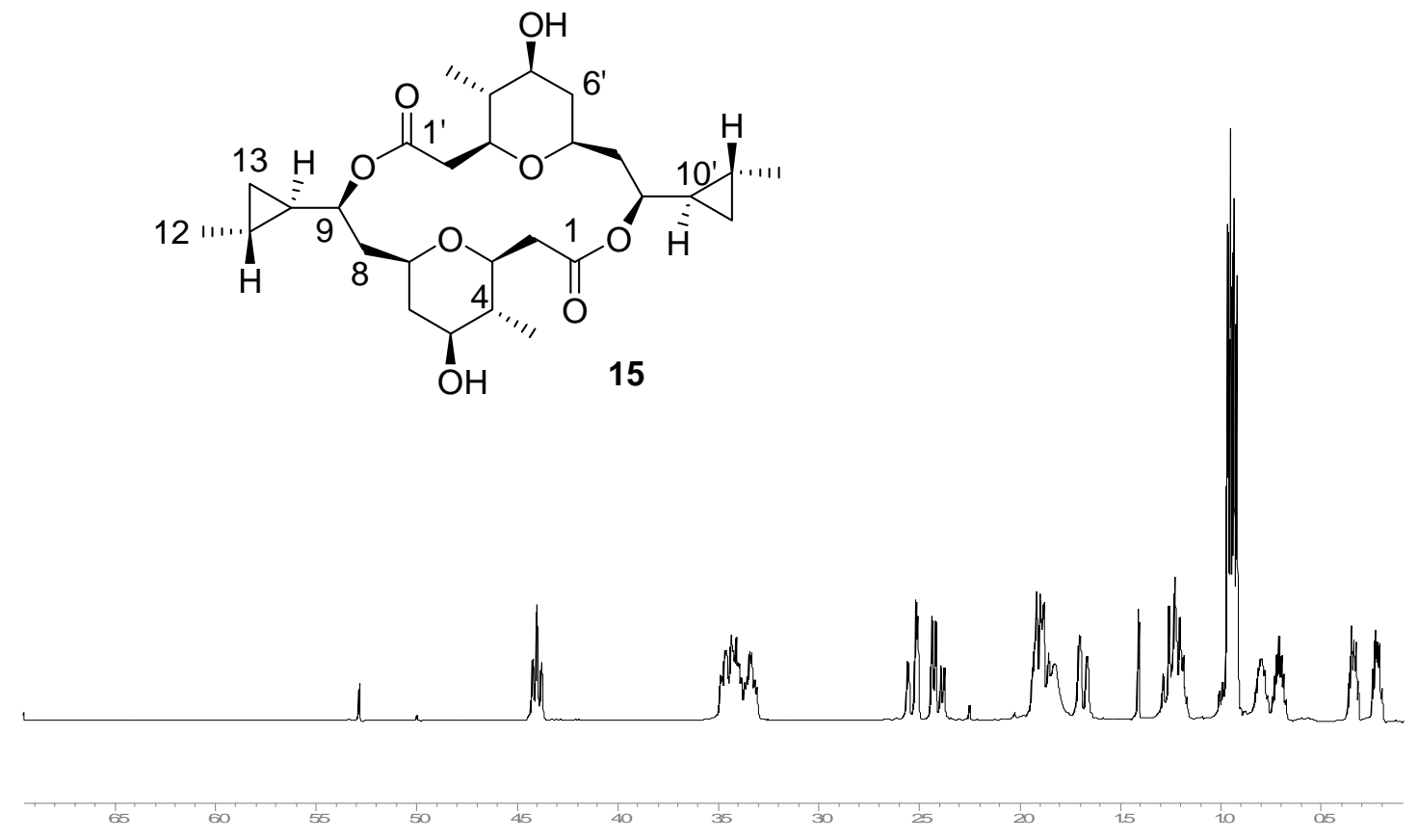

${ }^{13} \mathrm{C}$ Aglycone 15. 


\section{Supporting Information}

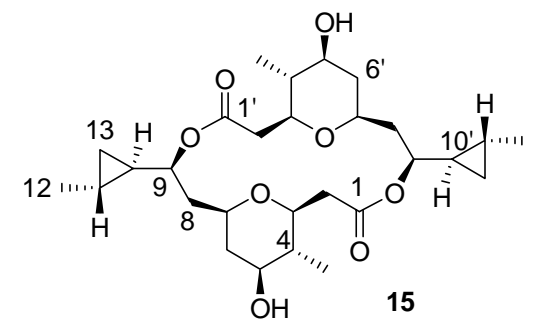

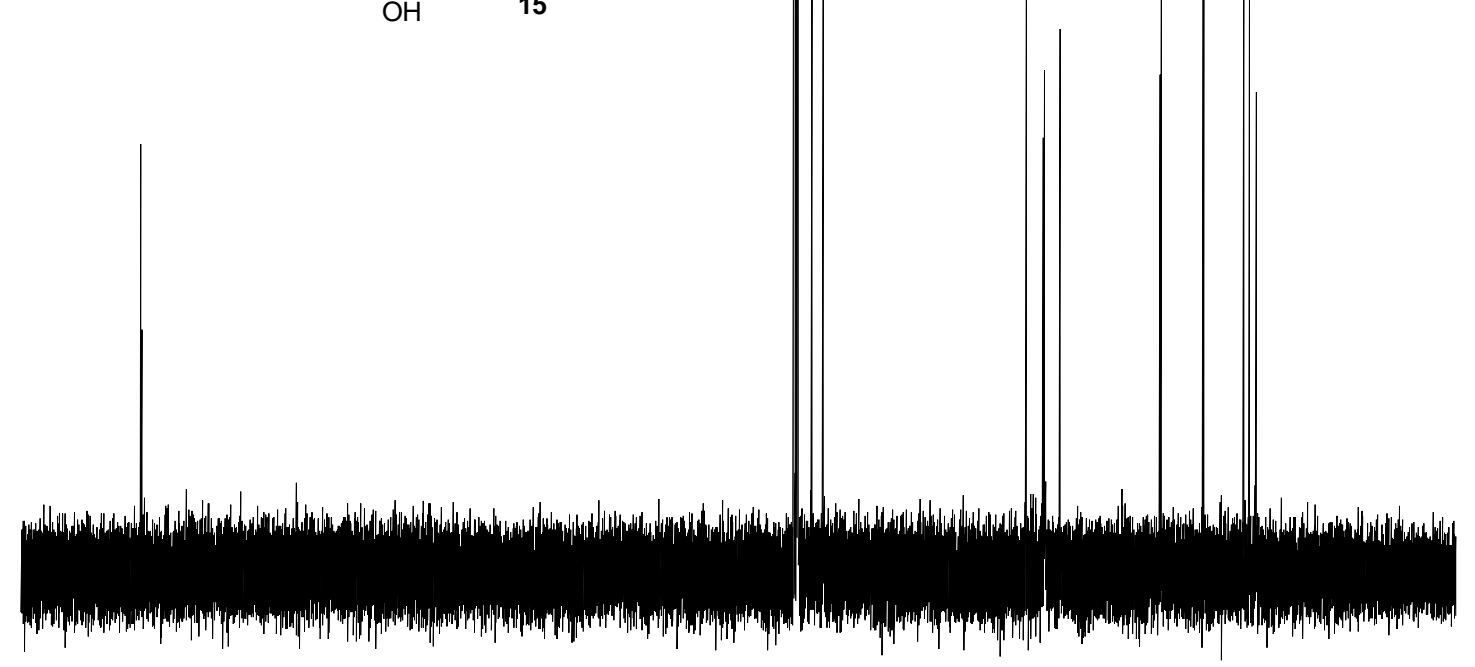

${ }^{1} \mathrm{H}$ Synthetic (-)-Clavosolide A 2.

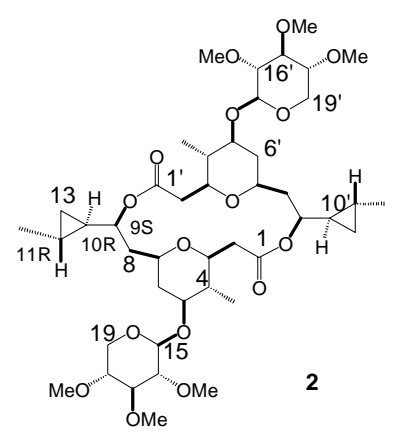

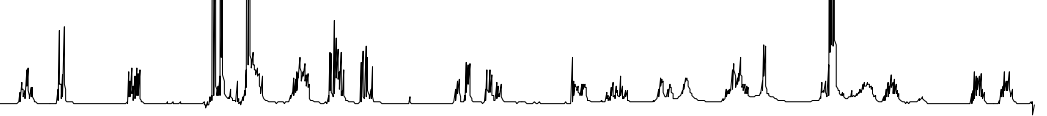

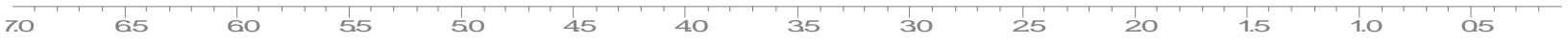




\section{Supporting Information}

${ }^{13} \mathrm{C}$ Synthetic (-)-Clavosolide A 2.
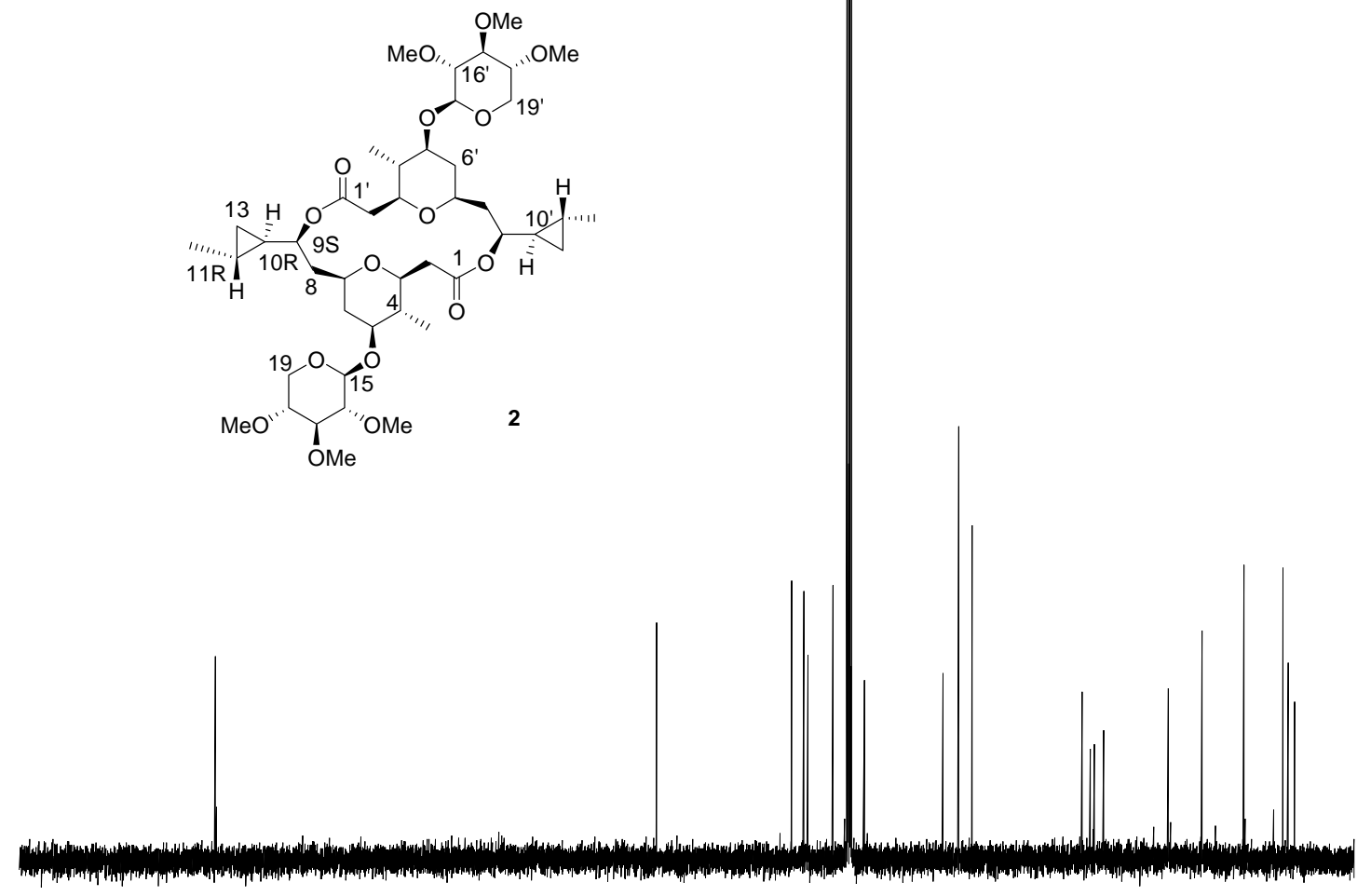